\title{
Whole genome mapping as a fast-track tool to assess genomic stability of sequenced Staphylococcus aureus strains
}

\author{
Julia S Sabirova, Basil Britto Xavier, Margareta leven, Herman Goossens and Surbhi Malhotra-Kumar*
}

\begin{abstract}
Background: Whole genome (optical) mapping (WGM), a state-of-the-art mapping technology based on the generation of high resolution restriction maps, has so far been used for typing clinical outbreak strains and for mapping de novo sequence contigs in genome sequencing projects. We employed WGM to assess the genomic stability of previously sequenced Staphylococcus aureus strains that are commonly used in laboratories as reference standards.
\end{abstract}

Results: S. aureus strains ( $n=12$ ) were mapped on the Argus ${ }^{\mathrm{TM}}$ Optical Mapping System (Opgen Inc, Gaithersburg, USA). Assembly of Ncol-restricted DNA molecules, visualization, and editing of whole genome maps was performed employing MapManager and MapSolver softwares (Opgen Inc). In silico whole genome Ncol-restricted maps were also generated from available sequence data, and compared to the laboratory-generated maps. Strains showing differences between the two maps were resequenced using Nextera XT DNA Sample Preparation Kit and Miseq Reagent Kit V2 (MiSeq, Illumina) and de novo assembled into sequence contigs using the Velvet assembly tool. Sequence data were correlated with corresponding whole genome maps to perform contig mapping and genome assembly using MapSolver. Of the twelve strains tested, one (USA300_FPR3757) showed a 19-kbp deletion on WGM compared to its in silico generated map and reference sequence data. Resequencing of the USA300_FPR3757 identified the deleted fragment to be a $13 \mathrm{kbp}$-long integrative conjugative element ICE6013.

Conclusions: Frequent subculturing and inter-laboratory transfers can induce genomic and therefore, phenotypic changes that could compromise the utility of standard reference strains. WGM can thus be used as a rapid genome screening method to identify genomic rearrangements whose size and type can be confirmed by sequencing.

Keywords: Staphylococcus aureus, Deletion, ICE6013, Whole genome mapping, Whole genome sequencing, USA300

\section{Background}

The plasticity of the bacterial genome is well-known and can be attributed to horizontal gene transfer, genome rearrangements, and the activities of mobile genetic elements (MGEs) [1]. In the case of Staphylococcus aureus, and also for several other pathogens, MGE DNA accounts for a substantial part of the identified inter-strain genetic variability in terms of acquisition of virulence, colonization or antibiotic resistance factors. Such MGEassociated changes have also been reported in frozen, archived S. aureus strains [2], and are especially problematic

\footnotetext{
* Correspondence: surbhi.malhotra@uantwerpen.be

Department of Medical Microbiology, Vaccine \& Infectious Disease Institute, Universiteit Antwerpen, Antwerp, Belgium
}

for maintenance of reference culture collections utilized for inter- and intra-laboratory experimental validations.

Whole genome (optical) mapping (WGM) is an advanced molecular technology by which endonuclease-digested single DNA molecules are assembled into a high-resolution restriction map constituting a detailed genomic fingerprint. Originally developed in 1993 by Schwartz and colleagues to type Saccharomyces cerevisiae strains [3], WGM has so far mainly been applied in clinical microbiology for typing outbreak strains [4-7], and for genome sequencing to guide $d e$ novo assembly of sequence contigs [8]. Here, we applied WGM to rapidly assess genomic stability of key reference strains of Staphylococcus aureus utilizing their previously published whole genome sequences as comparators. 


\section{Results and discussion}

We utilized WGM to assess the genomic stability of previously sequenced $S$. aureus that are also commonly used as laboratory reference strains (Additional file 1: Table S1). Comparison of experimental whole genome maps to in silico restriction maps ( $\mathrm{NcoI}$ ) generated from published sequences showed that for majority of the strains (COL, MRSA476, 71193, ED133, JKD6008, JKD6159, LGA251, MRSA252, N315, TCH1516, and HO 5096 0412), both maps were identical (100-99.9\% map similarity; Figure 1). The $0.1 \%$ difference in in silico and experimental maps of some strains (COL, MRSA476, 71193, ED133, JKD6008, LGA251, MRSA252, N315, and TCH1516) could be attributed to the loss of small DNA fragments in the experimental maps. The rate of loss of fragments $\leq 2 \mathrm{~kb}$ could be as high as $75 \%$ during mapcard processing comprising in situ restriction, staining and washing of linearized and immobilized DNA molecules, as described previously [9]. However, for S. aureus FPR3757, a clear 1.5\% map difference was observed between the experimental and corresponding in silico WGMs. The experimental WGM of FPR3757 displayed a 19-kbp genomic deletion compared to its in silico map (Figure 2). According to published sequence data, the deleted fragment contained genes SAUSA300_1456 to SAUSA300_1474 that included a maltose degradation operon (Figure 3A).

In order to confirm the genomic deletion detected by WGM, the entire genome of FPR3757 was sequenced, de novo assembled into sequence contigs by Velvet with an optimized $k$-mer [10] and compared to its corresponding published genome using MapSolver. Re-sequencing of FPR3757 showed that the deleted fragment was $13354 \mathrm{bp}$ long (nucleotides 1630722 and 1644075) and comprised genes SAUSA300_1477 to SAUSA300_1489 (Figure 3B). The observed difference in prediction of the site of deletion by WGM and sequencing is likely to be due to the loss of small fragments during preparation of whole genome maps. Indeed, upon inspection of the FPR3757 map, the total number of restriction fragments was found to be remarkably lower than in the in silico map (219 and 244 fragments, respectively). Loss of fragments shorter than $2 \mathrm{~kb}$ can result in a certain degree of imprecision in prediction of the size of restriction fragments and localization of $\mathrm{NcoI}$ sites in the whole genome map, as compared to the in silico-generated map.

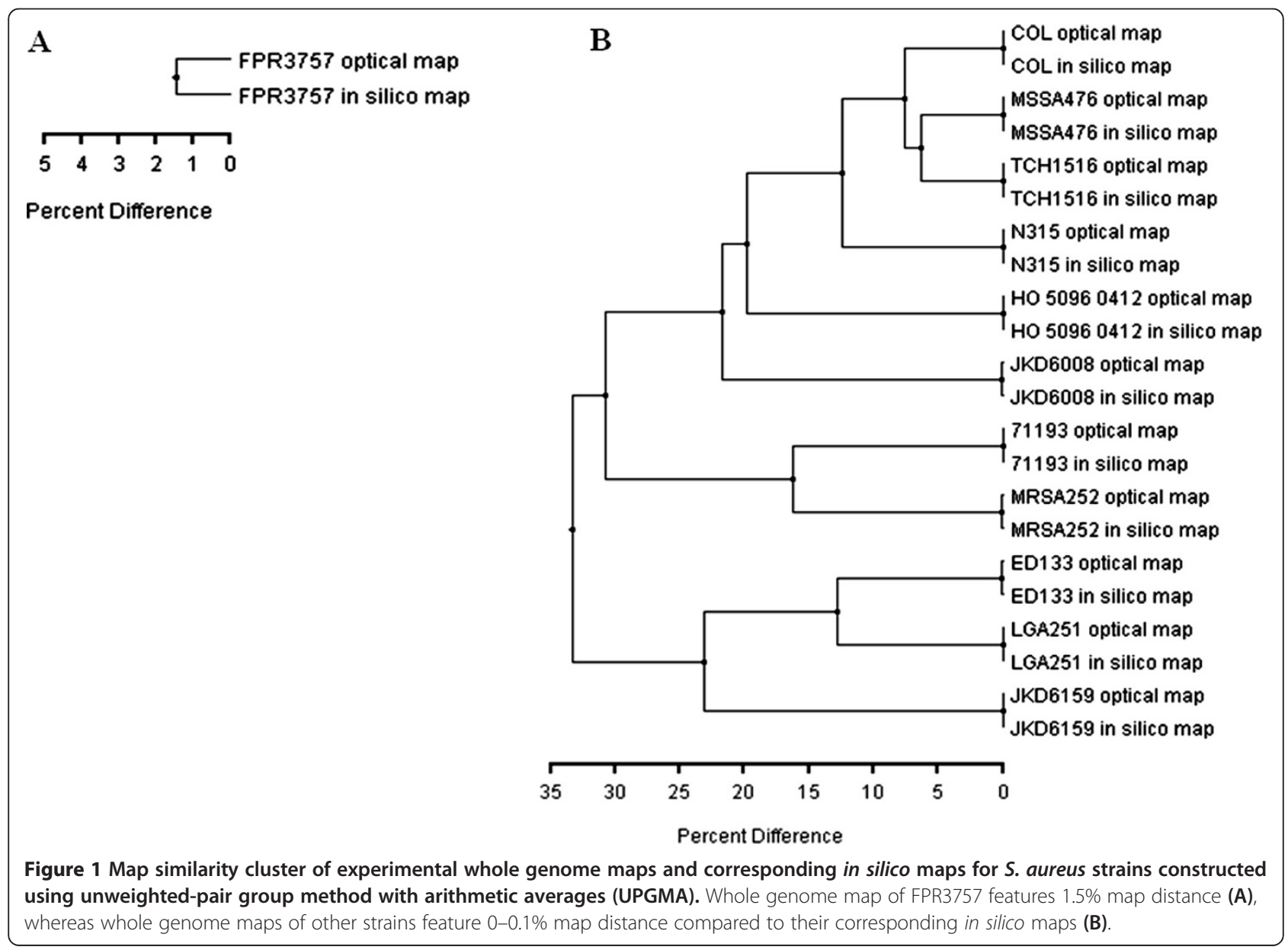




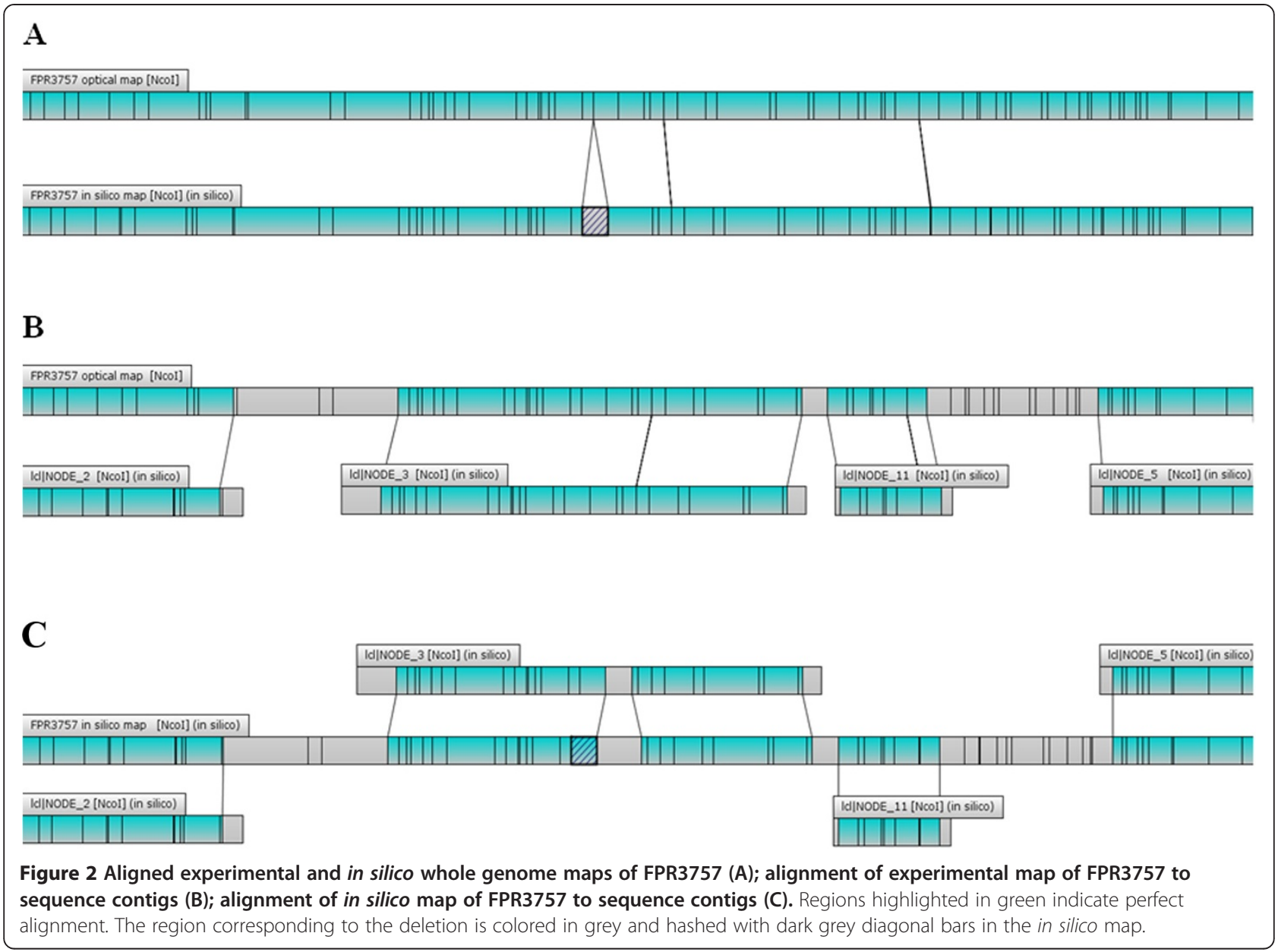

BLAST searches against $S$. aureus published genomes using the nucleotide sequence of the deleted fragment as a query identified an integrative conjugative element ICE6013, which was recently described as a novel MGE in a number of MRSA [11]. The MGE in FPR3757 showed 99\% pairwise identity to the ICE6053 of S. aureus strain HDG2 (accession number FJ231270), except for the absence of a 6551-bp Tn552 transposon. Comparison of the two ICEs employing blast2go and RAST functional comparison tools allowed to assign putative functions to the genes and to compare their genetic contents: the absence of Tn552 in the ICE in FPR3757 resulted in the loss of a $\beta$-lactamase operon comprising blaI, blaR1 and blaZ genes, whereas other genes typical for ICEs and potentially responsible for their transposition, replication and conjugative transfer were found to be intact $[12,13]$ (Figure 4, Additional file 2: Table S2).

\section{Conclusions}

In conclusion, WGM of sequenced S. aureus strains detected a large genomic deletion in one out of twelve strains studied here. Subsequent genome sequencing of the altered genome and inspection of the region identified the exact size, genomic location and the nature of this genomic deletion. WGM can thus be employed to rapidly assess genomic stability of strains over time during storage or after inter-laboratory transport followed by corroboration of genomic changes by whole genome sequencing.

\section{Methods}

\section{Bacterial strains and growth conditions}

Twelve S. aureus strains that are commonly used as reference strains and whose whole genome sequences are available in public databases were requested from strain collections (Network on Antimicrobial Resistance in S. aureus (NARSA, http://www.beiresources.org/), or from research groups: COL (Acc.no. NC_002951), 71193 (Acc.no. NC_017673), FPR3757 (Acc.no. NC_007793), ED133 (Acc.no. NC_017337), JKD6008 (Acc.no. NC_017341), JKD6159 (Acc.no. NC_017338), LGA251 (Acc.no. NC_017349), MRSA252 (Acc.no. NC_002952), MRSA476 (Acc.no. NC_002953), N315 (Acc.no. NC_002745), TCH1516 (Acc.no. NC_010079), and HO 50960412 (Acc.no. NC_017763). Upon arrival, bacterial stocks were inoculated on blood agar plates and incubated overnight at 


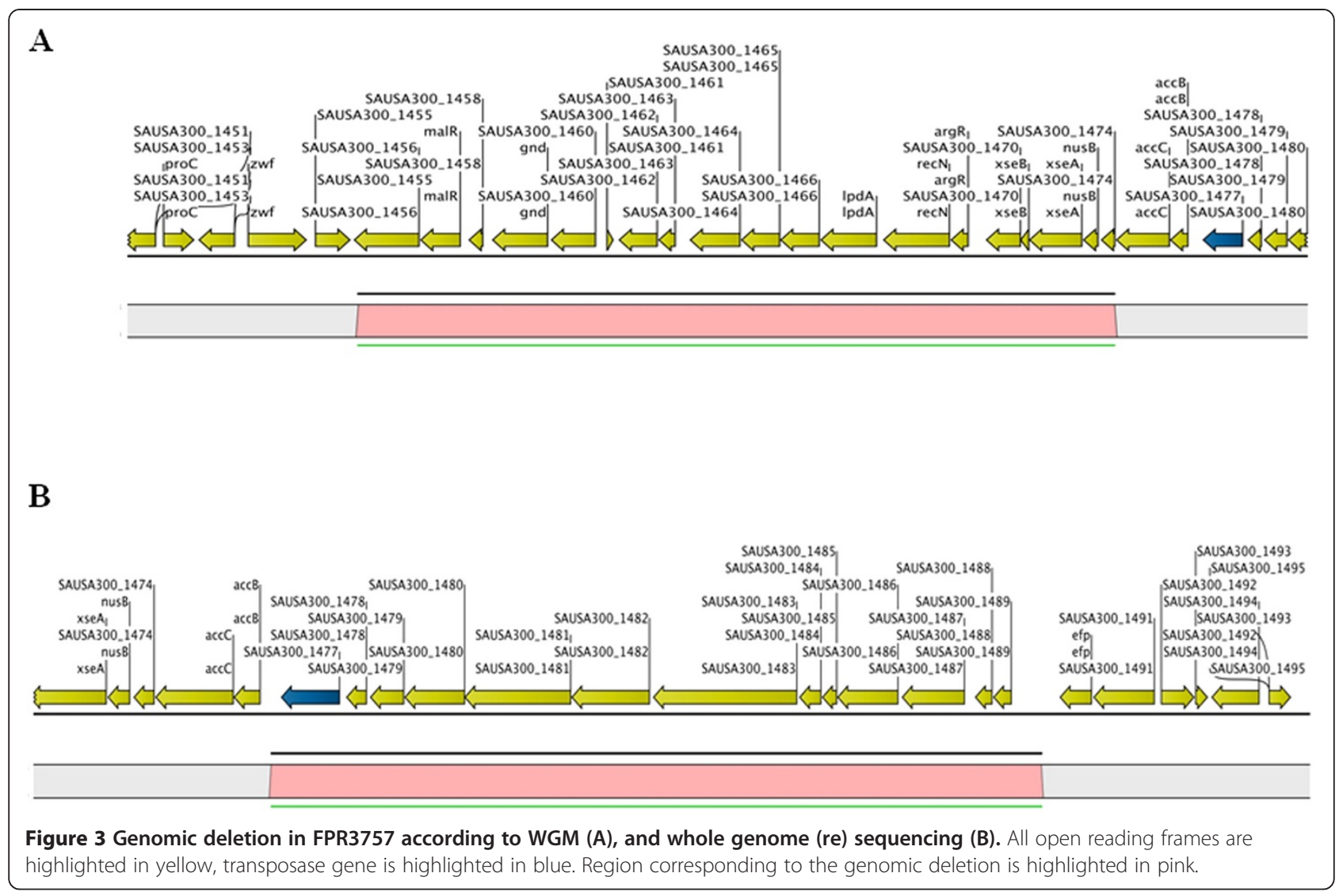

$37^{\circ} \mathrm{C}$. 16 hour-old colonies were used for species reconfirmation by Maldi-TOF and to prepare glycerol stocks for long-term storage at $-80^{\circ} \mathrm{C}$. In parallel, one of the 16 hour-old colonies was inoculated on brain heart infusion agar, incubated overnight at $37^{\circ} \mathrm{C}$ and used for WGM.

\section{Genome sequencing}

S. aureus strains were grown on brain heart infusion plates for $24 \mathrm{~h}$ at $37^{\circ} \mathrm{C}$ and used to extract genomic DNA using MasterPure ${ }^{\text {ma }}$ Gram Positive DNA Purification Kit (Epicentre Technologies Corp). Samples were prepared and sequenced employing Nextera XT DNA Sample Preparation Kit and Miseq Reagent Kit V2 (Illumina Inc). The $2 \mathrm{X} 150$ reads were de novo assembled into contigs using CLC Genomics Workbench with default parameters and Velvet v1.2.10 assembler [14] with an optimised N50 k-mer size of 93 [10]. Sequencing data were correlated with corresponding whole genome maps to perform contig mapping in MapSolver software (Opgen Inc). Gene annotation of ICE elements was performed using RAST annotation server and blast2go for functional annotation.

\section{Whole genome mapping}

Maps were generated on an Argus ${ }^{\mathrm{Tm}}$ Optical Mapping System (Opgen Inc, Gaithersburg, USA). DNA extraction, quality control, restriction using $\mathrm{NcoI}$, and loading on a mapcard were done according to manufacturer's protocols. Briefly, S. aureus colonies grown overnight at $37^{\circ} \mathrm{C}$ on brain heart infusion plates were used for isolation of high molecular weight (HMW) DNA using Argus $^{\ominus}$ HMW DNA isolation kit (Opgen, Inc). Extracted DNA preps were checked for the presence of HMW DNA molecules by using Argus ${ }^{\circ}$ QCard kit (Opgen, Inc) and subsequently used for mapping employing Argus ${ }^{\circ}$ MapCard II kit (Opgen, Inc). The assembly of restricted DNA molecules and identification of novel NcoI restriction sites was performed employing MapManager software (Opgen Inc.). Visualization and editing of maps was performed using MapSolver software (Opgen Inc.). For map editing, whole genome maps were adjusted in size, orientation and in starting point employing in silico maps generated from corresponding genome sequence data using MapSolver's function "Comparative genomics" and an advanced parameter "Allow alignments to wrap circular maps" with other default parameters pre-set in MapSolver. 


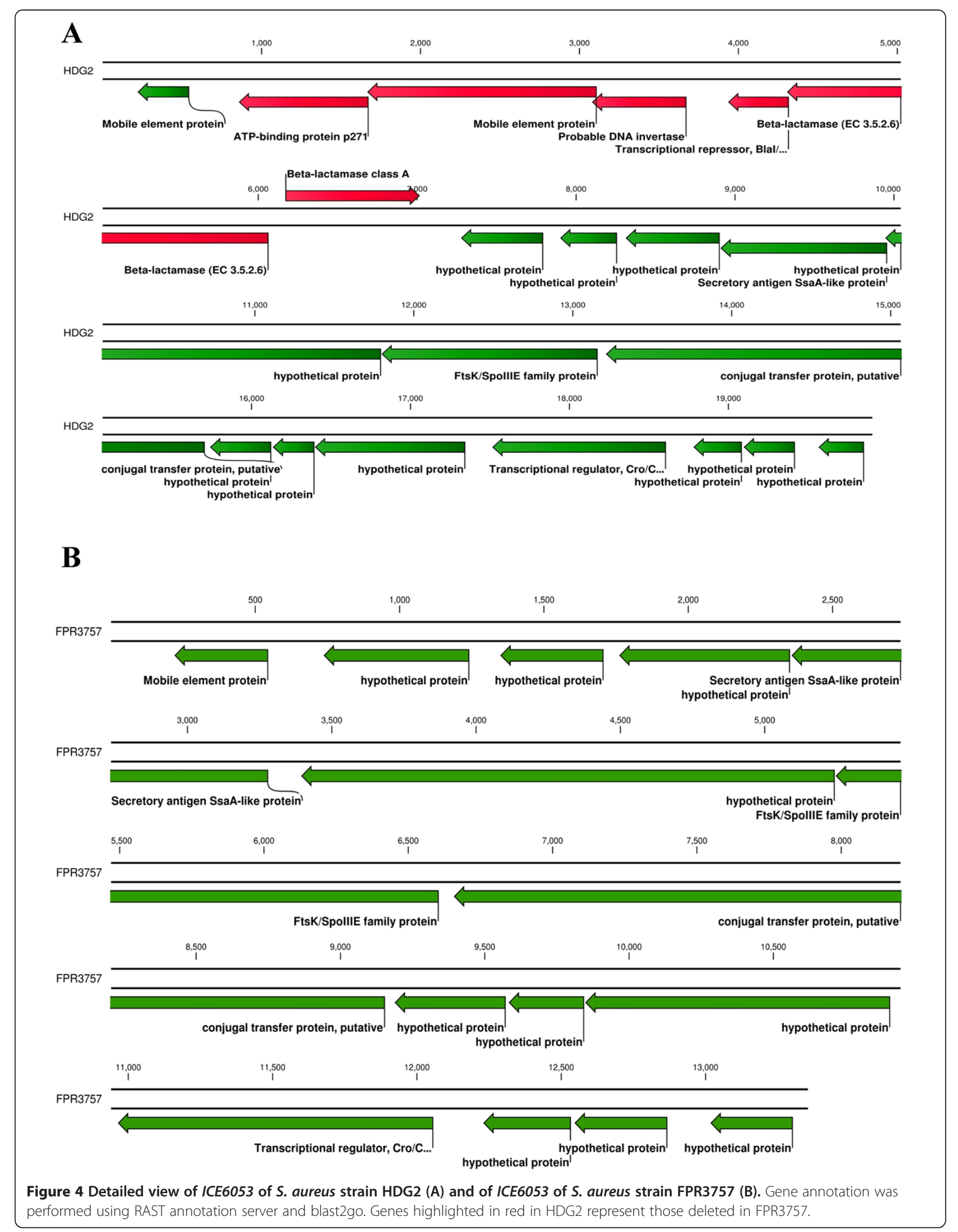


Map clustering was also performed using MapSolver's default parameters employing MapSolver's function "Create cluster".

\section{Additional files}

Additional file 1: Table S1. Staphylococcus aureus strains used in this study.

Additional file 2: Table S2. List of genes predicted in ICE6053 of S. aureus strain FPR3757 by RAST annotation server and blast2go.

\section{Abbreviations}

WGM: Whole genome mapping; ICE: Integrative conjugative element: MGE: Mobile genetic element; BLAST: Basic local alignment search tool; NARSA: Network on Antimicrobial Resistance in S. aureus.

\section{Competing interests}

The authors declare that they have no competing interests.

\section{Authors' contributions}

This study was designed by JSS, SM-K, HG, and MI. Experimental work was done by JSS and BBX. The manuscript was drafted by SM-K and JSS and was reviewed by all authors. All authors read and approved the final manuscript.

\section{Acknowledgements}

The authors are grateful to the following scientists for providing S. aureus strains: Frank Lowy and Sheetal Trivedi (The Columbia University), Ross Fitzgerald and Cartwright Robin (The University of Edinburgh), Benjamin Howden, Timothy Paul Stinear, Kirstie Mangas (University of Melbourne), Mark Holmes and Gavin Paterson (University of Cambridge). The authors also thank Prof. Jean-Pierre Hernalsteens for useful discussions and the Network on Antimicrobial Resistance in Staphylococcus aureus Program (NARSA) for providing other S. aureus isolates used in this study.

\section{Funding}

This work is supported by funding from Research Foundation Flanders (FWO-F, Research project no. G.0983.10N), and the European Community (PREPARE network contract FP7-HEALTH F3 2013- no. 602525). BBX is supported by University of Antwerp Research funds (BOF-DOCPRO 2012-27450).

Received: 13 June 2014 Accepted: 2 October 2014

Published: 8 October 2014

\section{References}

1. Darmon ELD: Bacterial genome instability. Microbiol Mol Biol Rev 2014, 78(1):1-39.

2. Hiramatsu K, Sukuzi E, Takayama H, Katayama Y, Yokota T: Role of penicillinase plasmids in the stability of the mecA gene in methicillinresistant Staphylococcus aureus. Antimicrob Agents Chemother 1990, 34(4):600-604.

3. Schwartz DC, Li X, Hernandez LI, Ramnarain SP, Huff EJ, Wang YK: Ordered restriction maps of Saccharomyces cerevisiae chromosomes constructed by optical mapping. Science 1993, 262:110-114.

4. Kotewicz ML, Mammel MK, Le Clerc JE, Cebula TA: Optical mapping and 454 sequencing of Escherichia coli 0157: $\mathrm{H} 7$ isolates linked to the US 2006 spinach-associated outbreak. Microbiology 2008, 154(11):3518-3528.

5. Jackson SA, Kotewicz ML, Patel IR, Lacher DW, Gangiredla J, Elkins CA: Rapid genomic-scale analysis of Escherichia coli 0104:H4 by using high-resolution alternative methods to next-generation sequencing. Appl Environ Microbiol 2012, 78:1601-1605.

6. Petersen RF, Litrup E, Larsson JT, Torpdahl M, Sørensen G, Müller L, Nielsen EM: Molecular characterization of Salmonella Typhimurium highly successful outbreak strains. Foodborne Pathog Dis 2011, 8(6):655-661.

7. Johnson PD, Ballard SA, Grabsch EA, Stinear TP, Seemann T, Young HL, Grayson ML, Howden BP: A sustained hospital outbreak of vancomycinresistant Enterococcus faecium bacteremia due to emergence of vanB $E$. faecium sequence type 203. J Infect Dis 2010, 202(8):1278-1286.
8. Onmus-Leone F, Hang J, Clifford RJ, Yang Y, Riley MC, Kuschner RA, Waterman PE, Lesho EP: Enhanced de novo assembly of high throughput pyrosequencing data using whole genome mapping. PLOS One 2013, 8(4):e61762.

9. Reslewic S, Zhou S, Place M, Zhang Y, Briska A, Goldstein S, Churas C, Runnheim R, Forrest D, Lim A, Lapidus A, Han CS, Roberts GP, Schwartz DC: Whole-genome shotgun optical mapping of rhodospirillum rubrum. Appl Environ Microbiol 2005, 71:5511-5522.

10. Xavier BB, Sabirova J, Pieter M, Hernalsteens JP, de Greve H, Goossens H, Malhotra-Kumar S: Employing whole genome mapping for optimal de novo assembly of bacterial genomes. BMC Res Notes 2014, 7:484.

11. Smyth DS, Robinson D: Integrative and sequence characteristics of a novel genetic element, ICE6013, in Staphylococcus aureus. J Bacteriol 2009, 191(19):5964-5975.

12. Arends K, Celik EK, Probst I, Goessweiner-Mohr N, Fercher C, Grumet L, Soellue C, Abajy MY, Sakinc T, Broszat M, Schiwon K, Koraimann G, Keller W, Grohmann E: TraG encoded by the pIP501 type IV secretion system is a two-domain peptidoglycan-degrading enzyme essential for conjugative transfer. J Bacteriol 2013, 195:4436-4444.

13. Parsons JA, Bannam TL, Devenish RJ, Rood Jl: TcpA, an FtsK/SpolllE homolog, is essential for transfer of the conjugative plasmid pCW3 in Clostridium perfringens. J Bacteriol 2007, 189(21):7782-7790.

14. Zerbino DR, Birney E: Velvet: algorithms for de novo short read assembly using de Bruijn graphs. Genome Res 2008, 18(5):821-829.

doi:10.1186/1756-0500-7-704

Cite this article as: Sabirova et al:: Whole genome mapping as a fast-track tool to assess genomic stability of sequenced Staphylococcus aureus strains. BMC Research Notes 2014 7:704.

\section{Submit your next manuscript to BioMed Central and take full advantage of:}

- Convenient online submission

- Thorough peer review

- No space constraints or color figure charges

- Immediate publication on acceptance

- Inclusion in PubMed, CAS, Scopus and Google Scholar

- Research which is freely available for redistribution 